\title{
Physical Basis of Magnetic Resonance Spectroscopy and its Application to Central Nervous System Diseases
}

\author{
${ }^{1}$ Nicolás Fayed, ${ }^{2}$ Salvador Olmos, ${ }^{1}$ Humberto Morales and ${ }^{3}$ Pedro J Modrego \\ ${ }^{1}$ Magnetic Resonance Unit. Clinica Quirón. Zaragoza. Spain \\ ${ }^{2}$ University School of Engineering. Zaragoza \\ ${ }^{3}$ Neurology Department. Miguel Servet University Hospital. Zaragoza. Spain
}

\begin{abstract}
Magnetic Resonance Spectroscopy is based on the chemical shift property of the atom nuclei when a magnetic field is applied. This technique offers invaluable information about living tissues with special contribution to the diagnosis and prognosis of the central nervous system diseases. Concentration of several metabolites can be assessed in a reproducible manner by means of modern clinical scanners. $\mathrm{N}$-acetyl-aspartate is regarded as a neuronal marker and its levels reflect the neuronal density with significant decreases in degenerative disease such as Alzheimer's disease. Cholinecompounds reflect the cell's membrane turnover and degradation. Myo-inositol has emerged as a glial marker with increases in degenerative diseases. The major usefulness of MRS has been reported in brain tumors, degenerative disorders, myelination defects and encephalopathies. In this review we report the physical basis and the contribution of MR spectroscopy to the diagnosis and prognosis of several diseases of the Central Nervous System.
\end{abstract}

Kew words: Central nervous system diseases, magnetic resonance spectroscopy, pathophysiology

Brief history and physical basis of magnetic resonance imaging: Imaging human internal organs with exact and non-invasive methods is very important for medical diagnosis, treatment and follow-up as well as for clinical research. Today, one of the most important tools for this purpose is the MRI. MRI scanners are based on the discovery of the nuclear magnetic resonance (NMR) phenomenon that was detected independently by $\mathrm{Bloch}^{[1]}$ and Purcell ${ }^{[2]}$ in 1946. They discovered that an atomic nucleus with unpaired protons in a strong magnetic field rotates with a frequency depending on the strength of the magnetic field and the nature of the atom. If it is submitted to a radio frequency (RF) field of this particular frequency, which is the resonance frequency, it absorbs energy and when the RF field is removed this energy is emitted through an electromagnetic wave of the resonance frequency. For this discovery Bloch and Purcell were awarded the Nobel Prize in Physics in 1952.

The MR phenomenon was initially used mainly for studies of the chemical structure of substances. The first two-dimensional magnetic resonance (MR) images were reconstructed in 1973 by Lauterbur ${ }^{[3]}$. By introducing gradients in the magnetic field he made it possible to determine the origin of the emitted RFsignals. The same year, independently of Lauterbur, Mansfield and Grannell demonstrated the Fourier relationship between the spin density and the NMR signal acquired in the presence of a magnetic field gradient ${ }^{[4]}$. These discoveries were groundbreaking and led to the currently used application of MR in medical imaging. It also led to the Nobel Prize in Medicine for
Lauterbur and Mansfield in 2003. Even more Nobel Prizes ${ }^{[1]}$ have been attributed for discoveries in the field of MR imaging. Richard Ernst was awarded the Nobel Prize in chemistry 1991 for his contributions on further development of the methodology of high-resolution nuclear MR spectroscopy in 1975.

The MRI medical scanners have been available since 1980 and since then the use of MR scanners has rapidly increased, in 2002, there were approximately 22,000 in use worldwide and more than 60 million MRI examinations were performed. Compared with other imaging modalities MR has many advantages, first of all it is non-invasive and to present knowledge has no secondary effects. It provides an amazingly strong imaging contrast between tissues and it can, as we will see in this chapter, be adapted to image other physical phenomena.

Today, the most frequently used MRI method in medicine is the anatomical MRI designed to differentiate tissue structures. It is used for basically any part of the body, brain, knees, arms etc. Another more recent imaging method is the functional MRI (fMRI) for mapping of activation patterns in the brain. This is an important modality for better understanding of function. When a brain region is activated new energy must be transported to this region which leads to an increased blood flow in this part of the brain. This can be imaged by repetitive MR scans and detected by appropriate signal processing methods.

A brief description of the principle governing the generation of MRI is presented. The magnetic resonance phenomenon can be described by both 
classical and quantum mechanical approaches. In this paper, the classical approach is used for the task of simplicity, although NMR can be more accurately analyzed by quantum mechanics.

Physical principles of MRI: Protons, neutrons and electrons have an angular momentum known as spin. Each spin can have the values $\pm 1 / 2,3 / 2,5 / 2$. Since spins in atomic nuclei with pair number of protons will cancel each other, only atoms with an odd number of protons have a net spin, which is necessary for being NMR-active. The most typical nucleus to use in the NMR experiment is the hydrogen nucleus, ${ }^{1} \mathrm{H}$, that has the spin states $\pm 1 / 2$. Since the signal of one spin is impossible to measure, spins are in general considered as an ensemble and are described in terms of precession around a spin magnetization vector, $M$. When no external field is applied the spins are randomly distributed between the spin up $(+1 / 2)$ and spin down($1 / 2$ ) position and the net spin of the ensemble of spins therefore equals zero.

In the presence of an external magnetic field $\mathrm{B}_{0}(\mathrm{a}$ polarizing field), the spin magnetization vector $M$ will align itself with the field and the spins start processing around $\mathrm{B}_{0}$. The frequency of precession is the natural resonance frequency of the spin system. This resonance frequency is known as the Larmor frequency $\omega_{0}=\gamma B_{0}$ where $\gamma$ is the gyromagnetic ratio specific for each kind of nucleus. When considering a spin system we will define a laboratory frame in which $\mathrm{M}$ appears to be stationary and aligned with $\mathrm{B}_{0}$. The axis, along which $\mathrm{B}_{0}$ acts, is the longitudinal $\mathrm{z}$-axis and the plane orthogonal to the $\mathrm{z}$-axis is the transverse $\mathrm{xy}$-plane. For a more detailed description ${ }^{[5]}$.

To obtain a measurable signal from the experiment the system must absorb energy that can later be emitted and measured. The absorption of energy is made by exciting the system with another time-varying rotating magnetic field, an RF pulse $B_{1}$, acting perpendicular to $\mathrm{B}_{0}$ and oscillating with the Larmor frequency, $\omega_{0}$. The RF-pulse tilts $\mathrm{M}$ away from the $\mathrm{z}$-axis and $\mathrm{M}$ starts processing about the rotating $\mathrm{B}_{1}$-field. The tip-angle between $\mathrm{M}$ and the $\mathrm{z}$-axis is dependent on the duration of the RF-pulse. For the time, $\tau$, the angle is given by $\alpha=B_{1} \tau$. The cases $\alpha=90^{\circ}$ and $\alpha=180^{\circ}$ are the most commonly used in NMR-imaging and are called a $90^{\circ}$ pulse and $180^{\circ}$ pulse respectively. When the RF-pulse is removed, $\mathrm{M}$ will fall back to its initial position aligned with $\mathrm{B}_{0}$, this process is called relaxation. It is during the relaxation that the energy that has been added to the system by the RF pulse is emitted and produces a RF signal, the free induction decay (FID). This signal can be measured by an antenna or receiver coil and interpreted, as we will see later, to generate the image.

The relaxation process contains two kinds of relaxation: The spin-lattice (longitudinal) relaxation and the spin-spin (transverse) relaxation. The spin-lattice relaxation process involves the exchange of energy between the spin-system and the surroundings. The equilibrium state is when the magnetization vector $M$ is aligned with the longitudinal $\mathrm{B}_{0}$. The time for the system to reach equilibrium is the spin-lattice relaxation time and is described by the time $\mathrm{T}_{1}$. The spin-spin relaxation is the process where spins come to thermal equilibrium with themselves, this is also called the transverse relaxation and it is described by the time $T_{2}$. The difference in the physical properties of the different tissue types is reflected in the relaxation times. It is this mechanism that generates the contrast between different tissue types in imaging $\left(\mathrm{T}_{1}, \mathrm{~T}_{2}\right.$ etc $)$.

The problem of the evolution of the magnetization under the influence of the sum of a constant and a rotating field with simultaneous relaxation was first solved by Bloch $^{[3]}$. He proposed a set of equations, which describe how a spin system evolves,

$$
\begin{aligned}
& \frac{d M_{z}}{d t}=\frac{M_{0}-M_{x}}{T_{1}}+\gamma(\mathrm{M} \otimes \mathrm{B}) z \\
& \frac{d M_{x}}{d t}=\gamma(\mathrm{M} \otimes \mathrm{B})_{x}-\frac{M_{x}}{T_{2}} \\
& \frac{d M_{y}}{d t}=\gamma(\mathrm{M} \otimes \mathrm{B})_{y}-\frac{M_{y}}{T_{2}}
\end{aligned}
$$

Where $B=B_{0}+B_{1}$. The complete set of solutions of the above equation system in terms of the originals variables is given by

$$
\begin{aligned}
& M_{x}(t)=\exp \left(\frac{-t}{T_{2}}\right)\left(M_{x}(0) \cos \left(\omega_{0} t\right)+M_{y}(0) \cos \left(\omega_{0} t\right)\right) \\
& M_{y}(t)=\exp \left(\frac{-t}{T_{2}}\right)\left(M_{y}(0) \cos \left(\omega_{0} t\right)-M_{x}(0) \sin \left(\omega_{0} t\right)\right) \\
& M_{z}(t)=M_{z}(0) \exp \left(\frac{-t}{T_{1}}\right)+M_{z}(0)\left(1-\exp \left(\frac{-t}{T_{1}}\right)\right)
\end{aligned}
$$

The chemical shift: Other important NMR parameter that can distinguish spins in a particular environment are: the self-diffusion coefficient $\mathrm{D}$, the isotropic chemical shift $d$ and the hyperfine splitting $J$. In a real spin system, all nuclei atoms and molecules have associated electrons. If a magnetic field is applied, the surrounding electron clouds tend to circulate in such a direction as to produce a field, which opposes that applied, causing a small chemical shift. The nucleus experiences a total field

$$
B_{e f f}=B_{0}(1-d)
$$

Where $d$ is the shielding. This shielding perturbation results in a shift of the resonant frequency for nuclei in different environments and this resultant effect is very useful in NMR spectroscopy. The chemical shift may be expressed as

$\delta=\frac{f-f_{T M S}}{f_{T M S}} 10^{6}$ 
Where $\delta$ is in parts per million (ppm), $f$ is the resonant frequency of the species of interest and $f_{T M S}$ is the resonant frequency of a reference substance (TMS: tetramethylsilane). The effect of chemical shift is observed in images where more than one chemical is present. The value of $\square$ is very small, usually on the order of a few parts per million and is dependent on the local chemical environment in which the nucleus is located. Fat $\left(\mathrm{CH}_{2}\right)$ is a well-known example of a chemically dependent component, which is chemically shifted about a $3.35 \mathrm{ppm}$ in Larmor frequency from water $\left(\mathrm{H}_{2} \mathrm{O}\right)$ protons. A large range of $\delta$ values exist for biological objects giving rise to many resonant frequency range of a spin system can expressed as

$$
\left|\omega-\omega_{0}\right| \leq \frac{\omega_{\max }}{2}
$$

Image formation and k-space: As previously mentioned, the Larmor frequency is dependent on the external field, $\mathrm{B}_{0}$. By using a polarizing gradient field $\mathrm{G}_{\mathrm{z}}$ linearly variable along the $\mathrm{z}$-axis but constant in time, the Larmor frequencies will change depending on its position. Due to this effect, a slice selection can be made by letting $\mathrm{B}_{1}$ oscillate with different frequencies dependent on the choice of slice to excite. Linear field gradients along the $x$ and $y$-axis, $G_{x}$ and $G_{y}$, are applied to determine position in the transverse xy-plane. Generally, $G_{y}$ is first applied and introduces a phaseshift in the FID-signal dependent on the position along the y-axis. The phase-shift is due to the difference in frequency that varies with position. It is determined relative to the phase introduced by $\mathrm{B}_{0}, \gamma \mathrm{B}_{0} \delta$. The phase-shift is given by:

$\varphi=\gamma \delta \mathrm{G}_{\mathrm{y}} y$,

where is $\gamma$ the gyro-magnetic ratio, $\delta$ is the length of time over which $\mathrm{G}_{\mathrm{y}}$ is applied and $y$ the position.

When the field gradient is removed the frequencies will return to their initial value but the phase-shifts between nuclei remain at different positions on the $y$ axis. Then the field gradient, $G_{x}$ is applied and the frequencies will change again, dependent on their position along the $\mathrm{x}$-axis. It is normally during the application of $G_{x}$ that the signal is detected. The resulting signal after successively applying $G_{z}, G_{y}$ and $G_{x}$ corresponds to the Fourier transform of the transversal magnetization $\mathrm{M}_{\mathrm{xy}}$. In order to make the Fourier relation between the signal and the magnetization more obvious a reciprocal spatial frequency space, known as k-space, is introduced. The measured signal for a set of gradients, $G_{x}, G_{y}$ and $G_{z}$, produces a single line in k-space. Applying these gradients in different combinations leads to different samplings of k-space. Once k-space has been sampled the MR image is obtained by applying the inverse Fourier transform. The above description samples kspace for one slice along the z-axis at a time (Fig. 1), but several different techniques for sampling the 3D volume exists ${ }^{[5]}$.

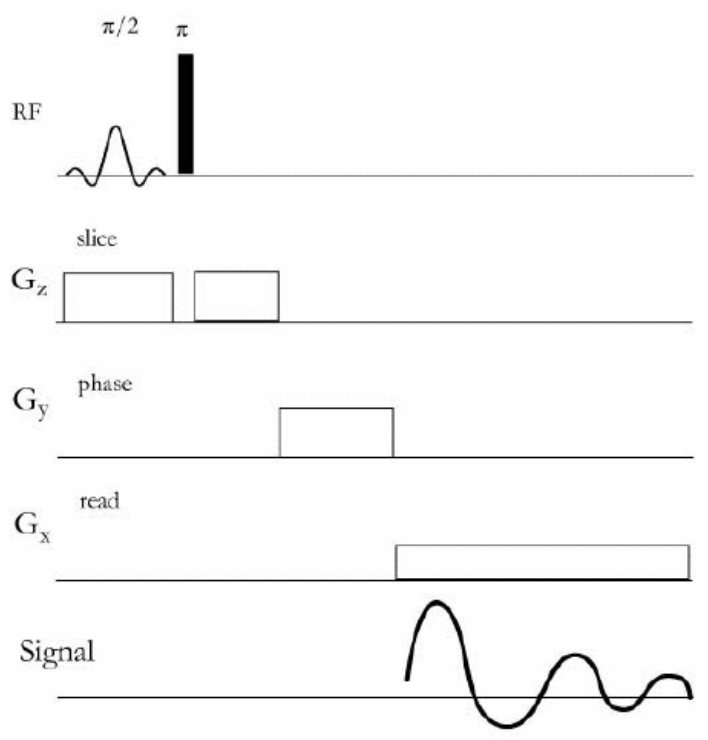

Fig. 1: Schematic figure of a typical imaging sequence for sampling $\mathrm{k}$-space. The z-gradient is responsible for the slice selection and the $\mathrm{x}$ and $y$-gradients are responsible for frequency and phase encoding respectively

\section{MAGNETIC RESONANCE SPECTROSCOPY}

By means of this technique we can study the chemical composition of living tissues. This technique is based on the chemical shift propriety of atoms. The concentration of some metabolites is determined from spectra that may be acquired in several ways.

Generally, two different approaches are used for proton spectroscopy of the brain: single-voxel methods based on the stimulated echo acquisition mode (STEAM) or point resolved spectroscopy (PRESS) pulse sequences and spectroscopy imaging (SI) (also known as chemical shift imaging (CSI) studies usually done in two dimensions using a variety of different pulse sequences (spin-echo (SE), PRESS).

The basic principle underlying single-voxel localization techniques is to use three mutually orthogonal slice selective pulses and design the pulse sequence to collect only the echo signal from the point (voxel) in space where all three slices intersect.

In STEAM, three $90^{\circ}$ pulses are used and the stimulated echo is collected. All other signals (echoes) should be dephased by the large crusher gradient applied during the so-called mixing time. Crusher gradients necessary for consistent formation of the stimulated echo and removal of unwanted coherences. In PRESS, the second and third pulses are refocusing $\left(180^{\circ}\right)$ pulses and crusher gradients are applied around these pulses to select the desired SE signal arising from all three RF pulses and dephasing unwanted coherences. STEAM and PRESS are generally similar but differ in a few key respects: 
* Slice profile (i.e. sharpness of edges of voxel): STEAM is somewhat better because it is easier to produce a $90^{\circ}$ pulse with a sharp slice profile than a $180^{\circ}$ pulse.

* SNR: Provided that equal volumes of tissue are observed and using the same parameters (repetition time (TR), TE, number of averages, etc.), PRESS should have approximately a factor of two better SNR than STEAM, because the stimulated echo is formed from only half the available equilibrium magnetization.

* Minimum TE: STEAM should have a shorter minimum TE than PRESS, since it uses a TM time period and shorter $90^{\circ}$ than $180^{\circ}$ pulses may be possible.

* Water suppression: STEAM may have slightly better water suppression factors, because water suppression pulses can be added during the TM period (this period does not occur in PRESS). Also, STEAM may have less spurious water signal from the $90^{\circ}$ slice selective pulses than the $180^{\circ}$ pulses in PRESS.

* Coupled spin systems and zero-quantum interference: The complex phenomena that can occur in coupled spin systems (e.g. Lac, Glu, etc), namely modulation of the echo signal by scalar couplings and/or the creation of zero-or multiplequantum coherences, may occur with both sequences. However, the detailed dependence of these compounds signal on $\mathrm{TE}$ and other experimental parameters will be different for STEAM and PRESS. STEAM is more susceptible for the creation of (usually unwanted) zeroquantum coherence because it uses $90^{\circ}$ pulses.

\section{APPLICATIONS OF MRS TO THE STUDY OF CENTRAL NERVOUS SYSTEM DISEASES}

MRS is a non-invasive method that provides metabolic/biochemical information about the brain. It has been widely used in daily practice. In this work, the technique is discussed and the most frequent applications of MRS are shown. We offer a practical approach to the method presenting the spectra of the most common neurologic entities. Our aim is to provide knowledge about MR spectroscopy not only to doctors but also to scientist interested in this field.

As a non-invasive method providing metabolic information about the brain, MRS enables tissue characterization on a biochemical level surpassing that of conventional magnetic resonance imaging (cMRI). MRS is also able to detect abnormalities that are invisible to cMRI, because metabolic abnormalities often precede structural changes. MRS does not replace cMRI but complements the information as a prognostic indicator, while following the progression of the disease and evaluating the response to treatment.
The most frequent used spectroscopy is that originated from Hydrogen nucleus (proton ${ }^{1} \mathrm{H}-\mathrm{MRS}$ ). This technique is based on the differences of resonance obtained from the hydrogen nuclei depending on the surrounding atoms (chemical shift). Each metabolite being assessed discloses different frequency of hydrogen resonance and appears in a different site of the spectrum. The most frequently evaluated metabolites are N-acetyl-aspartate (NAA), myo-inositol $(\mathrm{mI})$, choline $(\mathrm{Ch})$, creatine $(\mathrm{Cr})$ and glutamine $(\mathrm{Glx})$. The position of the metabolite signal is identified on the horizontal axis by its chemical shift, scaled in units referred to as parts per million (ppm). With the appropriate factors considered, such as the number of protons, the relaxation times and so forth, a signal can be converted to a metabolite concentration by measuring the area under the curve. As long as water is the main component of living beings and its concentration is much higher than that of metabolites, it becomes necessary to suppress the signal of resonance from the hydrogen of water ${ }^{[6,7]}$. A plot showing peak amplitudes and frequencies is obtained. Each spectrum shows peaks corresponding to the different metabolite values: Myo-inositol (mI), 3.56 and 4.06 ppm; Choline compounds (Ch), 3.23 ppm; Creatine $(\mathrm{Cr}), 3.03$ and 3.94 ppm; y N-acetil-aspartate (NAA), 2.02; 2.5 and 2.6 ppm; Glx-glutamine and glutamate, 2.1-2.55 ppm and $3.8 \mathrm{ppm}$. Ratios between metabolites and creatine are also of great value as they counteract the systematic errors of measurements. Other peaks observed are evaluated in visual way: lactate (Lac), 1.33 (peakdoublet) and 4.1(2nd peak) ppm; lipids (Lip), 0,8-1.3 ppm (Fig. 2). We also can see other peaks such as alanine at $1.48 \mathrm{ppm}$, Scylloinositol at $3.36 \mathrm{ppm}$, etanol (triplet resonance) at $1.16 \mathrm{ppm}$, macromolecules at 0.5 to $1.8 \mathrm{ppm}$ and acetate at $1.92 \mathrm{ppm}$.

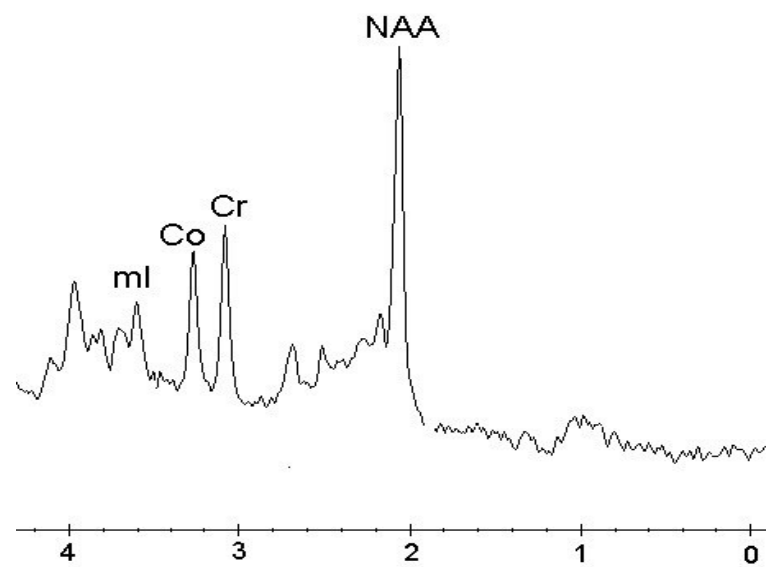

Fig. 2: Example of spectrum from the brain cortex with the different metabolite peaks

Myo-inositol has been regarded as glial marker located in the astrocytes, as product of myelin degradation and the most important osmolyte or cell volume regulator. Choline is a marker of the 
phospholipid metabolism and cellular membrane turnover marker, reflecting cellular proliferation. Creatine is used as internal reference value, since it is the most stable cerebral metabolite. It has a role in the energetic system of the brain and in the osmoregulation. NAA is a marker of neuronal and axonal viability and density. Lactate peak indicates anaerobic glycolysis in tumors. Lipids peak indicates necrosis and/or disruption of myelin sheath ${ }^{[8]}$.

Currently the spectra may be acquired with univoxel (SV) or multivoxel techniques. SV technique is readily available on most scanners. Voxels must be positioned away from sources of susceptibility artifacts and lipids. For diffuse processes, a 2 × 2 × $2 \mathrm{~cm}(8 \mathrm{~cm} 3)$ voxel is routinely used. For local lesions, the $\mathrm{SV}$ can be reduced in volume. Univoxel technique has the advantages of better spatial location, more homogeneity, better water suppression and quicker. However only one spectrum can be get per acquisition. Conversely, multivoxel technique (MV) makes it possible to obtain simultaneously multiple spectra per acquisition and to assess a greater area of the brain but with smaller spectral resolution. Up to date the univoxel technique is still superior to MV technique on the grounds of reproducibility ${ }^{[9-12]}$. For both SV and MV techniques, the MR scanner employs a process known as shimming to narrow peak linewidths within the spectra. For SV studies, improving the field homogeneity is performed with basic, zero-ordered shimming on clinical MR scanners. For MV, shimming to simultaneously produce uniform field homogeneity in multiple regions requires higher order shimming. To have high-quality spectra, we should avoid blood products, air, fat, necrotic areas, cerebrospinal fluid, metal, calcification and bone. In such areas differing magnetic susceptibility results in a non-homogenous field that hinders the production of diagnostic quality spectra.

With regard to the mode of acquisition, PRESS can be performed with short and long TE and there is complete recovery of signal. STEAM can be performed with very short echo times (TE), but there is incomplete recovery of signal and a precise volume element (voxel) is formed. The PRESS mode is more used than STEAM because it increases the signal/noise ratio and is less sensitive to movement artifacts ${ }^{[6]}$.

Echo Times have not yet standardized so far in MRS. In degenerative, demyelinating and vascular disease a short $\mathrm{TE}$ is advocated. In brain tumors the optimal TE is under debate. A short TE (20-40 ms) allows us to increase the signal/noise ratio and to visualize most metabolite peaks with the inconvenience of some degree of overlapping of peaks. Intermediate TE (135-144 ms) inverts the lactate peak to better distinguish it from lipids peak. Long TE (270-288 ms) gives worse signal/noise ratio but allows better visualization of some peaks (NAA, Choline and Creatine), because suppress the signal of others (myo- inositol, alanine, glutamate-glutamine $)^{[7,12,13]}$. Majós et $a l .^{[14]}$ demonstrated that a short TE yields better performance in the classification of tumors than long TE.

In the clinical practice time does matter so short TE are preferable $\mathrm{e}^{[12]}$. In our experience with a $1.5 \mathrm{~T} \mathrm{GE}$ Signa Horizon-clinical scanner a TE of $30 \mathrm{~ms}$ and a TR of $2500 \mathrm{~ms}$ have proven valuable ${ }^{[15]}$. Recently a TEaveraged PRESS technique yield highly simplified spectra with better suppression of signals not pertaining to the assessed metabolites such as that of macromolecules. TE is increased from $35 \mathrm{~ms}$ to $355 \mathrm{~ms}$ in steps of $2.5 \mathrm{~ms}$ with two acquisitions per step ${ }^{[16]}$.

\section{DEVELOPMENTAL DISORDERS}

In the newborn spectrum $\mathrm{mI}$ is the predominant metabolite whereas choline increases in the first days of life. NAA and creatine concentrations are lower than in adults. During the first weeks an increase of $\mathrm{Cr}$ and NAA is observed, as well as a decrease in $\mathrm{Ch}$ and $\mathrm{mI}^{[17]}$. Spectral abnormalities at this age can be of help for diagnosis and monitoring purposes, especially before myelination is completed.

In Table 1 and 2 are reported the spectroscopic values found in brain cortex and white matter in healthy individuals by age groups. NAA increases progressively with age in the white matter until the 20$25 \mathrm{~s}$, decreasing thereafter. In the cortex NAA decreases progressively from birth to old age.

Table 1: Normal spectroscopic values in the white matter of the centrum semiovale

\begin{tabular}{|c|c|c|c|}
\hline \multicolumn{4}{|c|}{ Mean age: 5 years ( range 3-9 years) n: 15} \\
\hline NAA: & 129.8 & $\mathrm{NAA} / \mathrm{Cr}$ & 2.03 \\
\hline Cr: & 73.5 & $\mathrm{Ch} / \mathrm{Cr}$ & $1.16 \Uparrow$ \\
\hline Ch: & $63.5 \Uparrow$ & $\mathrm{mI} / \mathrm{Cr}:$ & 0.68 \\
\hline & 42.7 & & \\
\hline \multicolumn{4}{|c|}{ Mean age: 14 years (range: $10-19$ years) n: 12} \\
\hline NAA: & 139.3 & NAA/Cr: & $2.26 \Uparrow$ \\
\hline Cr: & 67.6 & $\mathrm{Ch} / \mathrm{Cr}:$ & 0.86 \\
\hline Ch: & 58.7 & $\mathrm{mI} / \mathrm{Cr}:$ & 0.6 \\
\hline $\mathrm{mI}:$ & 36.3 & & \\
\hline \multicolumn{4}{|c|}{ Mean age: 36 years ( range: $20-52$ years) $n: 20$} \\
\hline NAA: & 124.2 & NAA/Cr: & 1.94 \\
\hline Cr: & 64.6 & $\mathrm{Ch} / \mathrm{Cr}:$ & 0.98 \\
\hline Ch: & 62.9 & $\mathrm{mI} / \mathrm{Cr}:$ & 0.65 \\
\hline
\end{tabular}

Table 2: Normal spectroscopic values in the brain cortex (Sample obtained in our center)

Mean age: 10 (range 7-16 years ) n:20

NAA: $124 \quad$ NAA/Cr: 1.90

$\begin{array}{llll}\text { Cr: } & 66 & & \\ \text { Ch: } & 48 & \mathrm{Ch} / \mathrm{Cr}: & 0.7\end{array}$

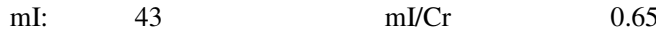

Mean age: 33 (range 20-38 years) n: 20

NAA: $115.8 \quad$ NAA/Cr: 1.82

$\begin{array}{llll}\text { Cr: } & 67.2 & \text { Cho/Cr: } & 0.65\end{array}$

Cho: $\quad 43.4 \quad \mathrm{mI} / \mathrm{Cr}: \quad 0.66$

mI: $\quad 43.9$

In congenital metabolopathies there may be specific and unspecific findings. 
In hyperammonemia glutamine is reportedly elevated as well as NAA in Canavan disease ${ }^{[8,17]}$. In familial leucodistrophies such as adrenoleucodystrophy and metachromatic leucodystrophy choline compounds may be elevated due to the presence of inflammation ${ }^{[7]}$.

In autistic children a decrease of NAA was reported in temporal cortex and cerebellar white matter ${ }^{[18]}$. In other studies with autistic children variable levels of $\mathrm{Ch}$ and $\mathrm{Cr}$ have been observed in different cortical areas ${ }^{[19]}$. WE did not see changes in the white matter of autistic children in the centrum semiovale in comparison to healthy controls. However we observed increased values of $\mathrm{NAA} / \mathrm{Cr}$ ratios in this area of children with attention-deficit/hyperactivity disorder ${ }^{[20]}$, which could suggest increased mitochondrial metabolism as it happens in the Asperger syndrome (autistic symptoms plus obsesive-compulsive behavior), or possibly to an increased synthesis of neurotransmitters. The interpretation of this finding is difficult because the exact function of NAA in the white matter remains elusive. In a small series of children with isolated developmental delay we found decreased $\mathrm{NAA} / \mathrm{Cr}$ ratios in the white matter in comparison to healthy children supporting the hypothesis of hypomielynation or delayed myelination $^{[21]}$.

Hypoxic encephalopathy: In the first year of life there is a significant correlation between spectroscopic values and neurological function with the lipids representing a metabolic marker of hypoxia. A decrease in the $\mathrm{NAA} / \mathrm{Cr}$ ratio indicates a grim prognosis. In neonatal hypoxia occipital cortex and basal ganglia are predominantly affected with decreased NAA and increased $\mathrm{mI}$, glutamate, lactate and lipids. Similar alterations are seen in the white matter ${ }^{[22]}$. Other affected cortical areas are the frontal and parietal ones ${ }^{[16]}$. Lipids peak is the main metabolic marker of hypoxia in the neonate. We have found cortical decreases of NAA and the presence of lipids and lactate in anoxic encephalopathy due to status epilepticus with radiological signs of laminar necrosis ${ }^{[23]}$.

In adults we can see interesting findings such as decreased creatine in the hippocampus of patients with sleep apnea ${ }^{[24]}$. In cardiorespiratory arrest decreases of NAA in cortex and cerebellun may be found with important prognostic value ${ }^{[25]}$.

Tumors: Usually we perform spectroscopy in tumors after contrast administration for better placement of the voxel, as long as gadolinium has no effect on metabolite levels ${ }^{[26-28]}$. It has proved useful for histologic grading purposes and for following the outcome after therapy. Choline-compounds (phosphoril-choline and glycerophosphoril-choline) are elevated in glial tumors in relation to accelerated cellular membrane turnover and degradation. Choline elevations can be seen also in pseudotumoral forms of multiple sclerosis, although in gliomas the aspartate levels are lower and perfusion better ${ }^{[29,30]}$.

Low levels of Creatine are inconstant and tend to indicate metastases from primitive tumors not containing creatine-kynase (kidney, lung, breast, lymphoma, prostate). Reductions or absence of NAA indicate the lack of neurons. This happens in tissues whose cells do not contain aspartate (gliomas, meningiomas, craneopharyngiomas and metastates). A typical meningioma is characterized by an absence of NAA, decrease of $\mathrm{Cr}$, prominent peak of choline and a peak of alanine and glutamine ${ }^{[31]}$.

Under normal conditions lactate is absent in the brain. It appears in cysts, necrotic tissues and in tissues with anaerobic glucolysis. Lipids peak indicates necrosis in malignant tumors either before or after treatment. Marked elevations of lipids are suggestive of lymphoma ${ }^{[32]}$. Elevation of mI, decrease of NAA and mild elevation of choline ${ }^{[33]}$ characterize low-grade gliomas (benign). High-grade gliomas (malignant) present with marked increase of choline, decrease of NAA and the presence of lactate and lipids peak ${ }^{[34-42]}$.

In our experience with gliomas and metastases, a $\mathrm{Ch} / \mathrm{Cr}$ ratio equal or higher than 1,56 and lactate peak predict malignancy at $88,9 \%$ sensitivity and $91,7 \%$ specificity ${ }^{[15]}$. In Fig. 3 is reported an example of spectrum corresponding to a malignant tumor.

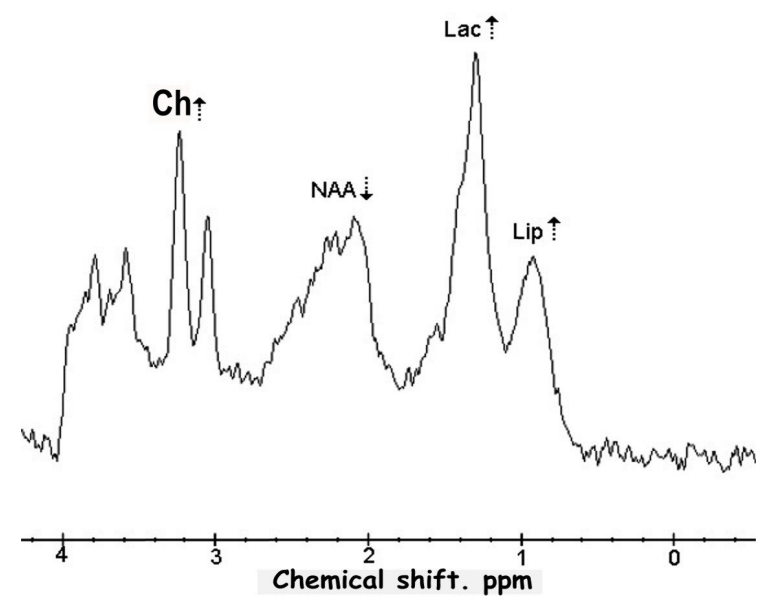

Fig. 3: Spectrum corresponding to glioblastoma with elevation of choline, decrease of NAA and lactate and lipids peaks

Radionecrosis: After chemotherapy and/or radiotherapy spectroscopic findings should be interpreted with caution during the first 6 months because radiotherapy elevates choline levels and there may be still tumoral cells. After 6 months choline elevation suggests tumoral recidive or therapy failure. In the event that no metabolites are found and lactate and lipids are present, then it suggests radionecrosis. Postradiotherapy demyelination appears after 6-8 months of treatment and may progress for two tears and 
its spectrum contains increases of choline compounds and $\mathrm{mI}$ due to gliosis and decreases of $\mathrm{NAA}^{[12]}$.

Neurocutaneous syndromes: In neurofibromatosis decrease of NAA and increase of $\mathrm{mI} / \mathrm{Cr}$ ratio have been observed and these findings are useful for monitoring of progression purposes. Norfray et al. (5) evaluated 19 patients with NF-1 and defined three spectral patterns based on the Cho/Creatine $(\mathrm{Cr})$ ratio, indicating hamartoma $(<1.5)$, undetermined lesion (transitional spectrum 1.5-2) and glioma (>2). In this study, $\mathrm{Cho} / \mathrm{Cr}$ ratio was less than 1.0 in control subjects.

Tuberous sclerosis may be significant elevations of $\mathrm{mI}$ on short echo proton MRS corresponding to gliosis, mild increase in choline and $\mathrm{N}$-acetyl aspartate (NAA) levels.

White matter disease: In mulltiple sclerosis the most common findings are decreased $\mathrm{NAA} / \mathrm{Cr}$ ratios and increased $\mathrm{Ch} / \mathrm{Cr}$ and $\mathrm{mI} / \mathrm{Cr}$ ratios. Progression of the disease is marked by a reduction of the NAA/Cr ratio. NAA is currently regarded as marker of axonal damage ${ }^{[43]}$. The active plaque may show the presence of lactate and lipids peak, as well as increased $\mathrm{Ch} / \mathrm{Cr}$ ratio and $\mathrm{mI}$. Inactive plaques show increased $\mathrm{mI} / \mathrm{Cr}$ ratio. In Schilder's disease, a rare demyelinating disease, we observed increased $\mathrm{Ch} / \mathrm{Cr}$ ratio, decreased $\mathrm{NAA} / \mathrm{Cr}$ ratio and the presence of lactate peak ${ }^{[44]}$.

Brain Ischemia: After a vascular occlusion a rapid elevation of lactate is detected with a trend to normalization after reperfusion. Conversely, there is a progressive decrease of NAA levels after ischemia with dramatic reductions or disappearance of NAA in the event of necrosis ${ }^{[45]}$. It has been postulated that the early changes seen in MRS could be a marker of ischemic penumbra when conventional MRI does not yield alterations and that it could be of help to better select patients for thrombolysis ${ }^{[46]}$.

Degenerative diseases: Most degenerative diseases share the fact of NAA decrease. In Alzheimer's disease the most frequent findings are the decrease of NAA levels and $\mathrm{NAA} / \mathrm{Cr}$ ratios as well as increases of $\mathrm{mI}$ and $\mathrm{mI} / \mathrm{Cr}$ ratios in cortical areas ${ }^{[47]}$. Furthermore this technique has proven useful to detect patients with isolated memory loss at high risk of conversion to Alzheimer's disease. A NAA/Cr ratio in the left parasagital occipital lobe predicted conversion at $100 \%$ sensitivity and $75 \%$ specificity, with an accuracy of classification of $88 \%{ }^{[48]}$. If these results are replicated MRS would become an important biomarker of the disease with implications in early detection and treatment.

Infectious diseases: Focal lesions showing a ring-shape contrast enhancement could generate problems of differential diagnosis between tumors and abscesses.
MRS has demonstrated to be a valuable tool for this purpose. Lipids and lactate peaks as well as elevations of several amino acids (leucine, isoleucine, valine, alanine) have been reported in bacterian abscesses and cysticercosis $^{[49,50]}$ (Fig. 4). In tuberculous abscesses only peaks of lactate and lipids are observed, without elevation of amino acids ${ }^{[51,52]}$.

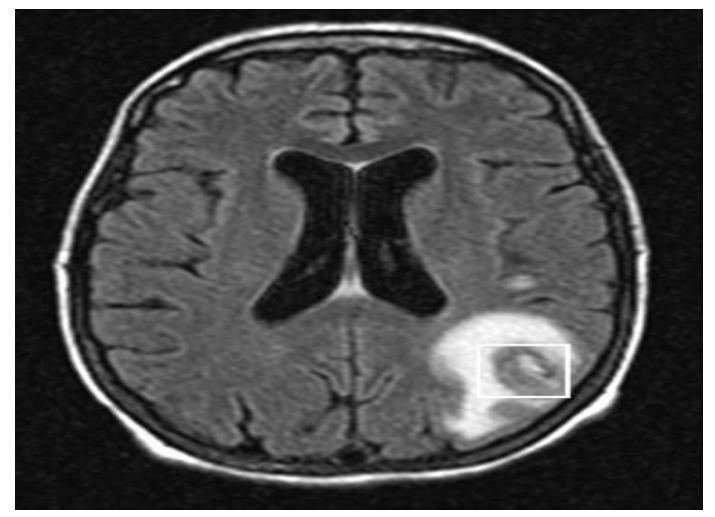

$4 \mathrm{~A}$

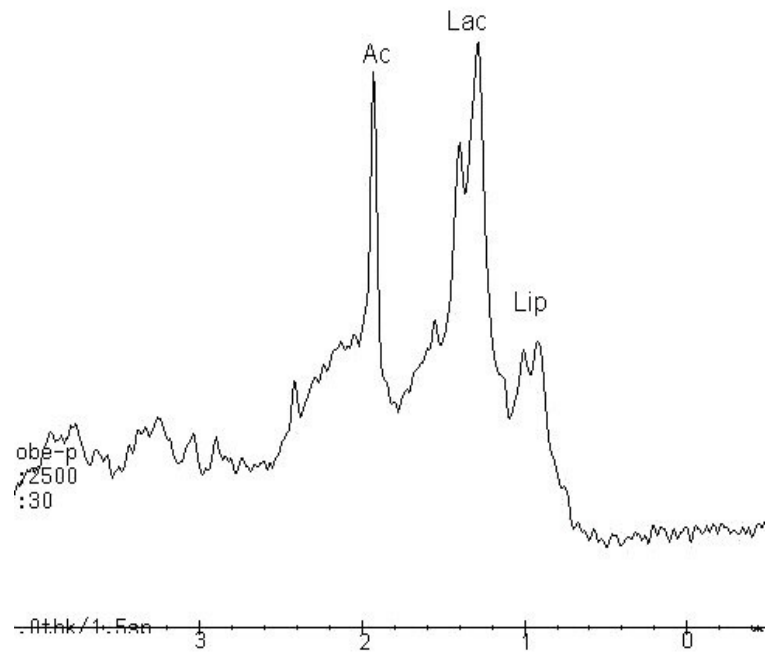

4B

Fig. 4: A: Computed Tomography of a bacterian abscess in the brain. B: Spectrum of the abscess with marked acetate peak (1.92 ppm), lactate (1.3 ppm) and lípids peak (0.8-1 ppm)

In the Reye's syndrome the main abnormality found on spectroscopy of the parietal white matter and/or occipital cortex is increased Glx levels, arising from glutamine and suggesting hyperammonibemiainduced encephalopathy. It is possible to quantify the Glx elevation by comparing Glx and NAA peak heights; if the Glx peak height is greater than one third of the NAA, there is an increase in Glx levels ${ }^{[53]} \mathrm{mI}$ levels might be reduced because of the osmolytic effects arising from hyperammoniemia. Lactate peak is common and there may be lipids.

In $\mathrm{HIV}+$ patients the NAA/Cr is decreased in AIDS cases in relation to patients without AIDS. These changes are seen even in patients with normal 


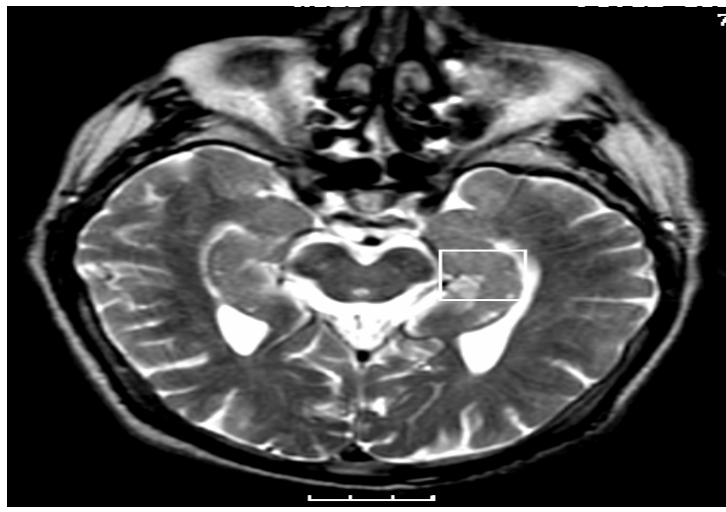

Fig. 5: Correct voxel-placement in the hippocampus

Table 3: Metabolite abnormalities by syndromes. Source. ${ }^{[11,14,49]}$

\begin{tabular}{|c|c|c|}
\hline Metabolite & Increased & Decreased \\
\hline Mio-inositol & $\begin{array}{l}\text { newborn, Azheimer } \\
\text { disease, diabetes } \\
\text { mellitus, } \\
\text { recovery from hypoxia, } \\
\text { hyperosmolar statel }\end{array}$ & $\begin{array}{l}\text { Hepatic } \\
\text { encephalopathy, stroke, } \\
\text { tumor, anoxyc } \\
\text { encephalopathy }\end{array}$ \\
\hline Choline compounds & $\begin{array}{l}\text { newborn, tumors, } \\
\text { trauma, diabetes, } \\
\text { chronic hipoxia, } \\
\text { hiperosmolar state, } \\
\text { adrenoleucodistrophy }\end{array}$ & $\begin{array}{l}\text { Hepatic } \\
\text { encephalopathy, stroke, } \\
\text { ementia }\end{array}$ \\
\hline Creatine & $\begin{array}{l}\text { Trauma, hiperosmolar } \\
\text { state, } \\
\text { aging }\end{array}$ & $\begin{array}{l}\text { Hipoxia, tumors, } \\
\text { stroke, newborn }\end{array}$ \\
\hline NAA & $\begin{array}{l}\text { Canavan disease, } \\
\text { Asperger syndrome, } \\
\text { Attention- } \\
\text { deficit/hyperactivity } \\
\text { disorder }\end{array}$ & $\begin{array}{l}\text { Developmental delay, } \\
\text { chilhood, hipoxia, } \\
\text { ischemia, herpes } \\
\text { encephalitis, epilepsia, } \\
\text { multiple sclerosis, } \\
\text { tumors, Alzheimer } \\
\text { disease, hemorrhage, } \\
\text { Alexander disease, } \\
\text { NPH, diabetes }\end{array}$ \\
\hline Lactate & $\begin{array}{l}\text { Hipoxia, stroke, } \\
\text { Canavan disease, } \\
\text { Alexander disease, } \\
\text { hemorrhage, } \\
\text { hydrocephalus, tumors, } \\
\text { abscesos }\end{array}$ & \\
\hline Lipids & Lymphomas, necrosis & \\
\hline
\end{tabular}

ADHD: attention-deficit/hiperactivity disorder; NPH: normal

pressure hydrocephalu

appearing brains on conventional MRI and could serve to anticipate appropriate treatment ${ }^{[54]}$. In HIVassociated pathologies of the brain abnormalities have also been described. MRS is especially useful in focal lesions ${ }^{[55]}$. In toxoplasmosis all metabolites are decreased (NAA, Cr, Ch and $\mathrm{mI}$ ), but marked peaks of lactate and lipids. In the CNS lymphoma NAA and $\mathrm{Cr}$ are decreased, $\mathrm{Ch}$ is increased with marked peaks of lactate and lipids. Progressive multifocal leucoencephalopathy tends to show low levels of NAA and $\mathrm{Cr}$ but high levels of $\mathrm{Ch}$ and $\mathrm{mI}$; there may be lipids and lactate peaks but less marked than in the other lesions. In cryptococal abscesses all of the metabolites are decreases but with the presence of lipids peak.

Temporal epilepsy: Correct voxel positioning, including most of the hippocampus is vital (Fig. 5).
Incorrect positioning (too anterior or posterior) may lead to susceptibility artifacts that disrupt metabolic ratios and consequently result in an inaccurate interpretation. NAA/Cho $+\mathrm{Cr}$ is the most useful parameter. The reduction in this ratio is considered pathologic if less than $0.71^{[56]}$. An asymmetry index of $=/>15 \%$ is used for lateralization of the epileptogenic focus, with very good correlation to electroencephalogram findings. Symmetric placement of voxels makes it possible to observe significant decrease of the NAA/Cr ratio in the damaged lobe.

In hippocampal sclerosis we can see reduction of NAA levels in relation to $\mathrm{Ch}$, lactate peak and either normal or increased $\mathrm{Ch} / \mathrm{Cr}$ ratio $^{[3]}$. It has been advocated that all epileptic syndromes in children should be evaluated with $\mathrm{MRS}^{[17]}$. In Table 3 are presented the main metabolite abnormalities according to the different syndromes ${ }^{[57]}$.

\section{CONCLUSION}

MRS has added important information to the understanding of pathophysiology of many CNS diseases. It has also demonstrated significant value for diagnostic purposes, especially for early diagnosis. As long as this technique is more widely used the contributions to the medical literature are increasing with more applications than expected years ago.

\section{REFERENCES}

1. Bloch, F., 1946. Nuclear induction. Phys. Rev., 70: 460-474.

2. Purcell, E.U., HC Torrey, RV Pound, 1946. Resonance absorption by nuclear magnetic moments in a solid. Phys. Rev., 69: 37-38.

3. Lauterbur, P.C., 1973. Image formation by induced local interactions: examples employing nuclear magnetic resonance. Nature, 242: 190-191.

4. Mansfield, P., and PK Grannell, 1973. Solid State Physics. J. Phys., 6: L422-426.

5. Callaghan, P.T., 1991. Principles of Nuclear Magnetic Resonance Microscopy. Oxford University Press. Oxford.

6. Maheshwari, S., G. Fatterpeaker, M. Castillo and S.K. Mukherji, 2000. Proton MR spectroscopy of the brain. Semin Ultrasound CT MR., 21: 434-451.

7. Bonavita, S., F. Di Salle and G. Tedeschi, 1999. Proton MRS in neurological disorders. Eur. J. Radiol., 30: 125-131.

8. Castillo, M., L. Kwock, and SK Mukherji, 1996. Clinical applications of proton MR spectroscopy. AJNR Am. J. Neuroradiol., 17: 1-15.

9. Sauter, R., M. Schneider, K. Wicklow and H. Kolem, 1991. Localized 1H MRS of the human brain: Single-voxel versus CSI techniques (abstract). J. Magn. Reson. Imaging, 1: 241. 
10. Hsu, Y.Y., C. Chang, C.N. Chang, N.S. Chu, K.E. Lim and J.C. Hsu, 1999. Proton MR spectroscopy in patients with complex partial seizures: singlevoxel spectroscopy versus chemical-shift imaging. AJNR Am. J. Neuroradiol., 20: 643-51.

11. Hsu, Y.Y., M.C. Chen, K.E. Lim and C. Chang, 2001. Reproducibility of hippocampal single-Voxel proton MR spectroscopy and chemical shift imaging. AJR Am. J. Roentgenol., 176: 529-36.

12. Law, M., 2004. MR spectroscopy of brain tumors. Top Magn. Reson. Imaging, 15: 291-313.

13. Burlina, A., T. Aureli, F. Bracco and F. Conti, 2000. MR spectroscopy: a powerful tool for investigating brain function and neurological diseases. Neurochem, Res., 25: 1365-1372.

14. Majós, C., M. Juliá-Sapé, J. Alonso and M. Serrallonga, et al., 2004. Brain tumor classification by proton MR spectroscopy: comparison of diagnostic accuracy at short and long TE. AJNR Am. J. Neuroradiol., 25: 1696-1704.

15. Fayed, N., H. Morales, P.J. Modrego and M.A. Pina, 2006. Contrast/Noise ratio on conventional MRI and Choline/Creatine ratio on proton MR spectroscopy accurately discriminate low-grade from high-grade cerebral gliomas. Acad. Radiol., 13: 728-737.

16. Hancu, I., E.A. Zimmerman, N. Sailasuta, R.E. Hurd, 2005. MR spectroscopy using TE averaged PRESS: A more sensitive technique to detect neurodegeneration associated with Alzheimer's disease. Magn. Reson. Med., 53: 777-782.

17. Ross, B. and T. Michaelis, 1994. Clinical applications of magnetic resonance spectroscopy. Magn. Reson. Q., 10: 191-247.

18. Otsuka, H., M. Harada, K. Mori, S. Hisoka and H. Nishitani, 1999. Brain metabolites in the hippocampus-amygdala region and cerebellum in autism: a 1H-MR spectroscopy study. Neuroradiology, 41: 517-519.

19. Levitt, J.G., J. O’Neill, R.E. Blanton, S. Smalley, D. Fadale and J.T. McCracken, et al., 2003. Proton magnetic resonance spectroscopic imaging of the brain in childhood autism. Biol. Psychiatry, 54:1355-1366.

20. Fayed, N. and P. Modrego, 2005. Comparative study of cerebral white watter in autism and attention-deficit/hyperactivity disorder by means of magnetic resonance spectroscopy. Acad. Radiol., 12: 566-9.

21. Fayed, N., H. Morales and P. Modrego et al., 2006. White matter proton MR spectroscopy in children with isolated developmental delay: Does it mean delayed myelination? Acad. Radiol., 3: 229-235.

22. Moats, R., L. Watson, T. Shonk, S. Tokuyama, D. Braslau and R. Eto et al., 1995. Added value of automated clinical proton MR spectroscopy of the brain. J. Comput. Assist. Tomogr., 19: 480-491.
23. Serrano, M., J.R. Ara, N. Fayed, R. Alarcia and A. Latorre, 2001. Hipoxic encephalopathy and cortical laminar necrosis. Rev. Neurol., 32: 843-847.

24. Bartlett, D.J., C. Rae, C.H. Thompson, K. Byth, D.A. Joffe and T. Enright, et al., 2004. Hippocampal area metabolites relate to severity and cognitive function in obstructive sleep apnea. Sleep Med., 5: 593-6.

25. Wartenberg, K.E., A. Patsalides and M.S. Yepes, 2004. Is magnetic resonance spectroscopy superior to conventional diagnostic tools in hypoxicischemic encephalopathy? J. Neuroimaging, $14: 180-6$.

26. Lin, A.P. and B.D. Ross, 2001. Short-echo time proton MR spectroscopy in the presence of gadolinium. J Comput Assist Tomogr., 25: 705712.

27. Murphy, P.S., A.S. Dzik-Jurasz, M.O. Leach and H. Rowland, 2002. The effect of Gd-DTPAon $\mathrm{T}(1)$-weighted choline signal in human brain tumours. Magn. Reson. Imaging, 20: 127-130.

28. Smith, J.K., L. Kwock and M. Castillo, 2000. Effects of contrast material on singlevolume proton MR spectroscopy. Am. J. Neuroradiol., 21: 10841089.

29. Law, M., D.E. Meltzer and S. Cha, 2002. Spectroscopic magnetic resonance imaging of a tumefactive demyelinating lesion. Neuroradiology, 44: 986-989.

30. Saindane, A.M., S. Cha, M. Law, X. Xue, E.A. Knopp and D. Zagzag, 2002. Proton MR spectroscopy of tumefactive demyelinating lesions. Am. J. Neuroradiol., 23: 1378-1386.

31. Shimizu, H., T. Kumabe, T. Tominaga, T. Kayama, K. Hara, Y. Ono et al., 1996. Noninvasive evaluation of malignancy of brain tumors with proton MR spectroscopy. Am. J. Neuroradiol., 17: 737-747.

32. Harting, I., M. Hartmann, G. Jost, C. Sommer, R. Ahmadi and S Heiland et al., 2002. Differentiating primary central nervous system lymphoma from glioma in humans using localized proton magnetic resonance spectroscopy. Neurosci. Lett., 342: 163166.

33. Castillo, M., J.K. Smith and L. Kwock, 2000. Correlation of mio-inositol levels and grading of cerebral astrocytomas. Am. J. Neuroradiol., 21: 1645-9.

34. Sabatier, J., V. Gilard, M. Malet-Martine, J.P. Ranjeva, C. Terral and S. Breil, 1999. Characterization of choline compounds with in vitro $1 \mathrm{H}$ magnetic resonance spectroscopy for the discrimination of primary brain tumors. Invest. Radiol., 34: 230-5.

35. Fan, G., B. Sun, Z. Wu, Q. Guo and Y. Guo, 2004. In vivo single-voxel proton MR spectroscopy in the differentiation of high-grade gliomas and solitary metastases. Clin. Radiol., 59: 77-85. 
36. Nelson, S.J., T.R. McKnight and R.G. Henry, 2002. Characterization of untreated gliomas by magnetic resonance spectroscopy imaging. Neuroimaging Clin. N. Am., 12: 599-613.

37. Moller-Hartmann, W., S. Herminghaus, T. Krings, G. Marquardg, H. Lanfermann and U. Pilatus, 2002. Clinical application of proton magnetic resonance spestroscopy in the diagnosis of intracranial mass lesions. Neuroradiology, 44: 371-381.

38. Law, M., S. Cha, E.A. Knopp, G. Johnson, J. Arnett, and A.W. Litt, 2002. High-grade gliomas and solitary metastases: differentation by using perfusion and proton spectroscopy MR imaging. Radiology, 222: 715-721.

39. Howe, F.A., S.J. Barton, S.A. Cudlip, M. Stubbs, D.E. Saunders and M. Murphy et al., 2003. Metabolic profiles of human brain tumors using quantitative in vivo $1 \mathrm{H}$ magnetic resonance spectroscopy. Magn. Reson. Med., 49: 223-232.

40. Majós, C., J. Alonso, C. Aguilera, M. Serrallonga, J. Perez-Martin and J.J. Acebes et al., 2003. Proton magnetic resonance spectroscopy of human brain tumors: assessment of differences between tumor type and its applicability in brain tumor categorization. Eur. Radiol., 13: 582-91.

41. Croteau, D., L. Scarpace, D. Hearshen, J. Gutierrez, J.L. Fisher and J.P. Rock et al., 2001. Correlation between magnetic resonance spectroscopy imaging and image-guided biopsies:semiquantitative and qualitative histopathological analysis of patients with untreated glioma. Neurosurgery, 49: 823-9.

42. Bulakbasi, N., M. Kocaoglu, F. Ors, C. Tayfun and T. Ucoz, 2003. Combination of single-voxel proton MR spectrocopy and apparent diffusion coefficient calculation in the evaluation of common brain tumors. Am. J. Neuroradiol., 24: 225-233.

43. De Stefano, N., L. Guidi, M.L. Stromillo, M.L. Bartolozzi and A. Federico, 2003. Imaging neuronal and axonal degeneration in multiple sclerosis. Neurol. Sci., 24: S283-6.

44. Iñiguez, C., L. Pascual, Y. Ramón, S. Cajal, N. Fayed and F. Morales, 2001. Transitional multiple sclerosis (Schilder's disease): A case report. J. Neurol., 247: 974-976.

45. Howe, F.A., R.J. Maxwell, D.E. Saunders, M.M. Brown and J.R. Griffiths, 1993. Proton spectroscopy in vivo. Magn. Reson. Q., 9:31.

46. Moseley, M.E., Y. Cohen, J. Mintorovitch, L. Chileuitt, H. Shimizu and J. Kucharczyk et al., 1990. Early detection of regional cerebral ischemia in cats: Comparison of diffusion and T2-weighted MRI and spectroscopy. Magn. Reson. Med., 14: 330-346.
47. Weiss, U., R. Bacher, H. Vonbank, G. Kemmler, A. Lingg and J. Marksteiner, 2003. Cognitive impairment: Assessment with brain magnetic resonance imaging and proton magnetic resonance spectroscopy. J. Clin. Psychiatry, 64: 235-42.

48. Modrego, P., N. Fayed and M.a. Pina, 2005. Conversion from mild cognitive impairment to probable Alzheimer's disease predicted by brain magnetic resonance spectroscopy. Am. J. Psychiatry, 162: 667-75.

49. Dev, R., R.K. Gupta, H. Poptani, R. Roy, S. Sharma and M. Husain, 1998. Role of in vivo proton magnetic resonance spectroscopy in the diagnosis and management of brain abscesses. Neurosurgery, 42: 37-43.

50. Pandit, S., A. Lin, H. Gahbauer, C.R. Libertin and B. Erdogan, 2001. MR spectroscopy in neurocysticercosis. J. Comput. Assist. Tomogr., 25: 950-952.

51. Gupta, R.K., D.K. Vatsal, N. Husain, S. Chawla, K.N. Prasad and R. Roy et al., 2001. Differentiation of tuberculous from pyogenic brain abscesses with in vivo proton MR spectroscopy and magnetization transfer MR imaging. Am. J. Neuroradiol., 22: 1503-1509.

52. Gupta, R.K., R. Roy, R. Dev, M. Husain, H. Poptani and R. Pandey et al., 1996. Finger printing of Mycobacterium tuberculosis in patients with intracranial tuberculomas by using in vivo, ex vivo, and in vitro magnetic resonance spectroscopy. Magn. Reson. Med., 36: 829-33.

53. Khan, T., M. Hollander and K. Baker et al., 2001. Clinical utility of short and ultra-short TE proton spectroscopy for evaluation of CNS infections. Presented at the 39th annual meeting of the American Society of Neuroradiology, Boston.

54. Vion-Dury, J., F. Nicoli and S. Confort-Gouny, 1994. Assessment of AIDS dementia complex by localized brain proton MRS. Proc. 11th European Society for Magnetic Resonance in Medicine and Biology, Vienna, Austria, pp: 454.

55. Chang, L., B.L. Miller, D. McBride, M. Cornford, G. Oropilla ans S. Buchthal et al., 1995. Brain lesions in patients with AIDS: H-1 MR spectroscopy. Radiology, 197: 525-531.

56. Achten, E., 1998. Aspects of proton MR spectroscopy in the seizure patient. Neuroimag. Clin. N. Am., 8: 849-862.

57. Danielsen, E. and B. Ross, 2001. Capítulo 72. Neuroespectroscopía. En Resonancia Magnética Stark D, Bradley W Jr. $3^{\text {a }}$ Ed. Mosby. 